\title{
How Well Does Neurology Residency Mirror Practice?
}

\author{
Fraser G.A. Moore, Colin Chalk
}

\begin{abstract}
Objective: To report the residency training experience of two neurologists, focusing on setting and diagnoses encountered. Methods: Data from prospective patient logs kept by the authors during residencies completed a decade apart were compared with each other and the literature. Results: The range of diagnoses was broadly similar between residencies, as were the proportions of common or uncommon disorders, and the proportion of cases without a neurological diagnosis. Although most patients were seen in in-patient settings, the rates at which common neurological conditions and functional disorders were seen was comparable to published experiences of community neurologists. Conclusions: The diagnostic profile of North American neurology residency appears to be relatively stable, regardless of location or date of training. In several respects, the content of current neurology residencies mirrors clinical practice well. Changes to residency training are doubtless needed, but they should be guided by a clear understanding of the experiences of contemporary trainees.
\end{abstract}

RÉSUMÉ: La résidence en neurologie reflète-t-elle bien la pratique? Objectif: Rapporter l'expérience de la formation en neurologie de deux neurologues en mettant l'accent sur le contexte et les pathologies observées. Méthodes: Les données recueillies de façon prospective par les auteurs pendant leur résidence effectuée à une décennie d'intervalle ont été comparées entre elles et à celles qui sont publiées. Résultats: La gamme des diagnostics était en général similaire ainsi que la proportion des pathologies fréquentes et rares rencontrées ainsi que la proportion de cas sans diagnostic neurologique. Bien que la plupart des patients aient été vus en milieu hospitalier, le taux de pathologies neurologiques fréquentes et de désordres fonctionnels était comparable à celui rapporté dans la littérature pour des neurologues en pratique courante. Conclusions: Le profile de la résidence en neurologie en Amérique du Nord semble être relativement stable quel que soit l'endroit ou le moment de la résidence. Sous plusieurs aspects, le contenu de la résidence en neurologie reflète bien la pratique clinique. Il ne fait aucun doute que des changements doivent être apportés à la formation en neurologie, mais ces changements devraient être guidés par une bonne compréhension de l'expérience des résidents actuels.

Can. J. Neurol. Sci. 2005; 32: 472-476

There is a growing conviction among those involved with neurology training that residency programs should increase the amount of time residents spend in the out-patient setting. ${ }^{1-4}$ Similar concerns have been expressed in other medical specialties $^{5,6}$ and in medical school. ${ }^{7}$ An important impetus for increasing out-patient exposure is to better equip trainees for post-residency clinical practice, by making residency more closely mirror clinical practice. However, there is a notable paucity of literature about the actual experience of a typical neurology trainee. The only detailed information about the kinds of patients seen, and the setting where seen (in-patient or outpatient), is from data collected by D'Esposito $^{8}$ during his neurology residency at Boston University in the 1980s. We present data on patient diagnoses compiled during residency by two neurologists, one trained in the United States in the late 1980s, and the other trained in Canada a decade later, and compare these with the experience of neurologists in practice.

\section{METHODS}

Unknown to each other, both authors had kept a prospective $\log$ of patients encountered during their residencies. In both logs, only new patients were included, meaning patients in whom a full neurological history and examination were performed for diagnosis of a new neurological problem. Follow-up encounters, patients seen during electro-diagnostic rotations such as electromyogram (EMG), and patients seen during rounds or presented at conferences were not included.

From the Department of Neurology and Neurosurgery, Centre for Medical Education, McGill University, Montreal, Quebec, Canada.

Received November 23, 2004. AcCePted in Final FORM June 2, 2005. Reprint requests to: Colin Chalk, Room L7-313, Montreal General Hospital, 1650 Cedar Avenue, Montreal, Quebec, Canada H3G 1A4. 
Colin Chalk's (CC) log covered the entire three years of neurology residency at Mayo Clinic, Rochester, Minnesota, from 1986 to 1989. For every patient in the log, age, sex, and neurological diagnosis were recorded prospectively. A total of 1009 patients were recorded (an additional 430 patients were examined in EMG lab rotations, but these are not included in the analysis below).

Fraser Moore's (FGAM) log began midway through the first of four years of core neurology training at McGill University, Montréal, from 1999 to 2002, and covered the last 39 consecutive months of the residency. For every patient, age, sex, neurological diagnosis, and location of the encounter (in-patient or out-patient) were recorded prospectively. A total of 1094 patients were collected (additional patients seen in EMG rotations were excluded).

For purposes of comparison, patient diagnoses were classified into 28 broad categories based on those used by D'Esposito. ${ }^{8}$ In addition, the proportions of common and uncommon disorders were determined. "Common" disorders were the three most frequent neurological disorders in Kurtzke's ${ }^{9} 1982$ US population survey, while "uncommon" disorders were those selected by D'Esposito ${ }^{8}$ : Huntington's disease, primary brain neoplasms, brain and spinal cord metastases, myasthenia gravis, and Guillain-Barré syndrome. The location of the patient encounters recorded by FGAM was compared with data reported by D'Esposito ${ }^{8}$ (These data were not recorded by CC). "Inpatients" were all patients seen on nursing units or in the emergency room and "out-patients" were all patients seen in offices or clinics.

\section{RESULTS}

Diagnoses: The Figure shows the ten most common diagnostic categories encountered by FGAM and CC, with the corresponding percentages reported by D'Esposito. ${ }^{8}$ (The Appendix contains details of all diagnoses recorded by the authors during their respective residencies). Noteworthy points are that in all three residencies, ischemic cerebrovascular disease was the most frequent diagnosis, and that ischemic cerebrovascular disease, headache, and epilepsy accounted for three of the top five diagnoses. The remaining top five diagnoses were peripheral neuropathy (in two residencies), metabolic encephalopathy, syncope, and Parkinson's disease. An important category not included in the Figure is patients whose symptoms were not felt to be due to a neurological disorder, which accounted for $16 \%$ of FGAM's patients and $14 \%$ of CC's. D'Esposito had included a category of "neurological symptoms," which encompassed $1.6 \%$ of his patients. These were patients whose symptoms were thought to be possibly neurological, but in whom a diagnosis was not established (D'Esposito, personal communication, 2003).

Common and uncommon disorders: The Table compares percentages of patients with common and uncommon neurological disorders seen during the residencies of FGAM, CC, and D'Esposito. ${ }^{8}$ The "common" disorders (stroke, headache, brain injury) are the top three neurological conditions identified in Kurtzke's 1982 US population survey. ("Brain injury" refers to closed head injury and includes conditions such as post-concussion syndrome). The "uncommon" disorders (primary brain neoplasm, brain and cord metastasis, Huntington's disease, myasthenia gravis, Guillain-Barre

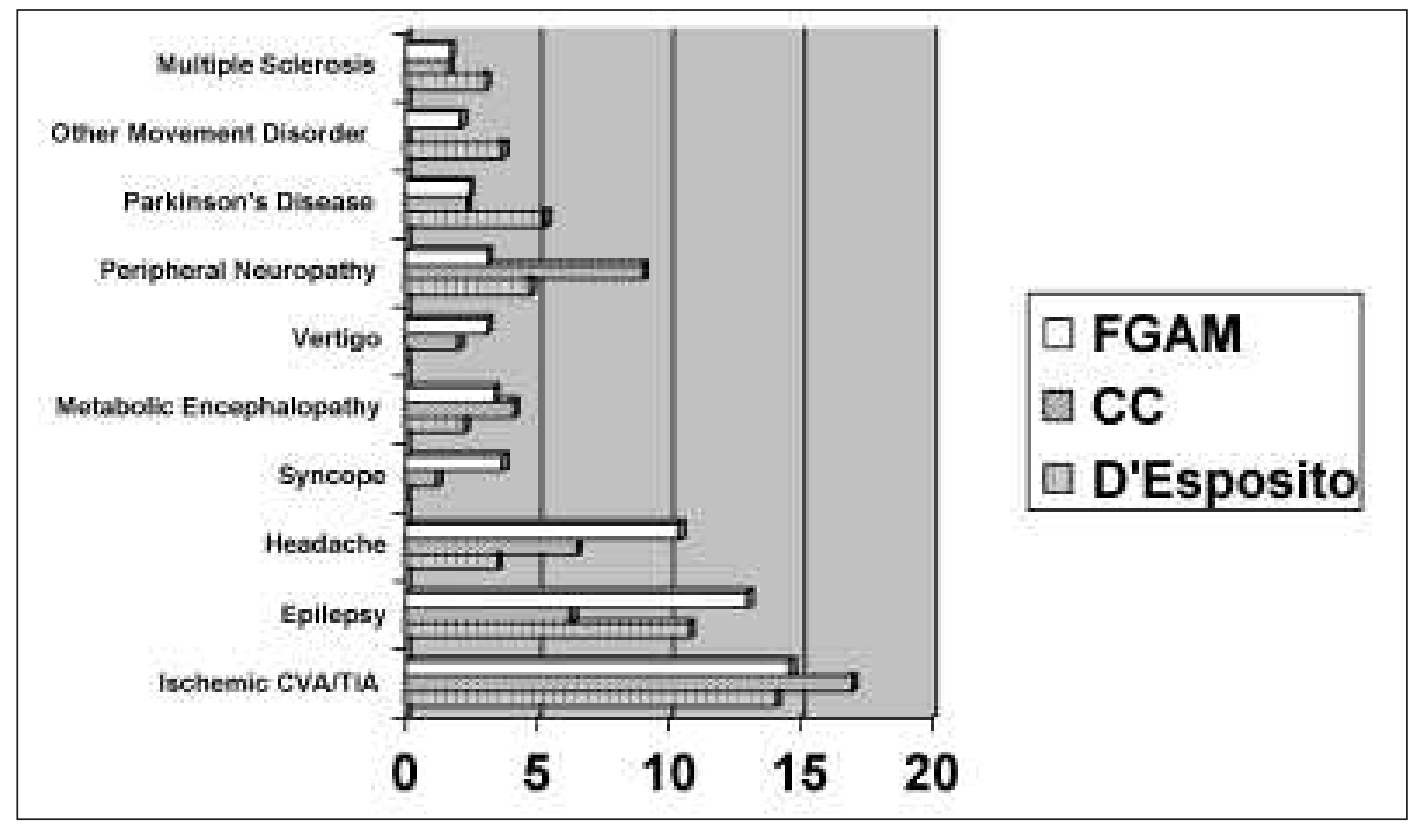

Figure: The ten diagnostic categories encountered most frequently by the first author (FGAM) during his residency. Data are expressed as a percentage of total new patients seen, and are compared to the corresponding percentages for the second author (CC) and D'Esposito. ${ }^{8} D$ 'Esposito's Huntington Disease and Essential Tremor categories are combined as Other Movement Disorders. $(C V A=$ cerebrovascular accident, TIA $=$ transient ischemic attack). 
Table: Comparison of the percentages of common and uncommon neurological diagnoses seen during the residencies of the authors (FGAM and CC) and D'Esposito. ${ }^{8}$ Common and uncommon diagnoses are those defined by D'Esposito, and are taken from Kurtzke's 1982 survey of the general US population. The percentage that these conditions contribute to the total neurological burden in the United States is also presented for comparison.

\begin{tabular}{l|l|l}
\hline Group & $\begin{array}{l}\text { Percentage of top 3 } \\
\text { neurological disorders* }\end{array}$ & $\begin{array}{l}\text { Percentage of uncommon } \\
\text { neurological disorders** }\end{array}$ \\
\hline FGAM & $26 \%$ & $3 \%$ \\
\hline CC & $25 \%$ & $10 \%$ \\
\hline D'Esposito $^{8}$ & $19 \%$ & $8 \%$ \\
\hline US Population & $53 \%$ & $0.4 \%$ \\
\hline
\end{tabular}

*stroke, headache, brain injury

**primary brain neoplasm, brain and cord metastasis, Huntington's disease, myasthenia gravis, Guillain-Barré syndrome

syndrome) are those selected by $\mathrm{D}^{\prime}$ Esposito $^{8}$ as examples of conditions relatively over-represented in residency training compared to their prevalence in the general population. The Table also presents the percentage of the total neurological burden in the United States for which these conditions accounted in Kurtzke's survey. ${ }^{9}$

Setting: Of the 1094 patients recorded by FGAM, 384 (35\%) were encountered in an out-patient setting, compared with 362 of $1332(27 \%)$ in D'Esposito's experience. ${ }^{8}$ The proportion of rotations within the residency specifically dedicated to outpatient clinics was 14\% for FGAM, versus D'Esposito's 6\%. In addition, FGAM spent one half day per week in out-patient clinics throughout his residency, regardless of rotation.

\section{Discussion}

Although much effort, past and present, has aimed to ensure that the content of neurology residency equips trainees to practice neurology safely and effectively, there is limited information about the clinical experiences of neurology residents. Our data, together with those of D'Esposito, give a detailed picture of the types of patients actually seen by residents in contemporary North American training. Among the noteworthy features of our data, perhaps most striking (and reassuring) is the relative similarity of patient profiles when comparing three residencies completed a decade apart, in three centers located in two different countries. Some local variation is seen between our data sets. Some of this might be anticipated, such as the abundance of Huntington's disease in D'Esposito's training in Boston, or the relative over-representation of peripheral neuropathy in a residency at Mayo Clinic, or of epilepsy during training at McGill.

Residency training is often held to over-emphasize exposure to rare disorders and to de-emphasize common conditions. The data presented in the Table could certainly be viewed as supporting this idea. However, a different perspective is gained if our data are compared to surveys of post-residency neurological practice. For example, surveys of Canadian neurological practices in Victoria (205 consecutive patients in a private office practice $)^{10}$ and Halifax (624 consecutive patients in a teaching hospital out-patient clinic) ${ }^{11}$ found that the "common" disorders listed in the Table accounted for $26 \%$ and $29 \%$ of patients, respectively, quite similar to the rates in the three residencies. A recent population-based study of neurological disorders seen by general practitioners in London, England found that the five conditions with the highest incidence were stroke/transient ischemic attack, shingles, diabetic polyneuropathy, compressive neuropathies, and epilepsy. ${ }^{12}$ This list is broadly similar to the top diagnoses in our Figure, although the UK study differs in important respects from ours, as patients were derived from general practice, and patients with headache and vertigo (which we encountered often) were specifically excluded.

Also noteworthy in our data sets was the large number of patient encounters in which no neurological disorder was thought to be present: $16 \%$ (FGAM) and 14\% (CC). Several studies have reported the prevalence of such patients (i.e., those with symptoms but without a discernable neurological disorder) in neurological practice. In the Victoria study, ${ }^{10}$ the "functional", "uncertain", and "skeletal" categories accounted for $18.5 \%$ of patients. "Functional" patients made up $11.2 \%$ of the Halifax cohort. "Undefined disorders" made up $7 \%$ of diagnoses in a cross-sectional study of neurologic practice in Colorado ${ }^{13}$ "symptoms without diagnosis" was one of the most common problems encountered in neurology practice in the Robert Wood Johnson Foundation Neurology Practice Study Report; ${ }^{14}$ and a survey of community neurological practice in the United Kingdom found that a specific diagnosis was not possible for $25 \%$ of referrals. ${ }^{15}$ Finally, in a survey of U.S. neurologists conducted by Rose, ${ }^{16} 13 \%$ of 1440 patients (20 consecutive patients seen by 72 neurologists) were diagnosed as having "anxiety", "neurosis", "depression", "no disease", or "paraesthesia". In other words, distinguishing between patients who have neurological disorders and those who do not is a 
significant part of neurological practice in different settings. Our data suggest that residency provides a significant and appropriate exposure to this type of patient, at a rate comparable to postresidency practice.

There is little doubt that the setting in which patients are seen in residency differs markedly from how most practicing neurologists spend their time. The experience of a neurologist practicing in Victoria, with almost $90 \%$ of time devoted to outpatients, ${ }^{10}$ is probably typical of most Canadian neurologists, and is certainly quite different from the experience of FGAM or D'Esposito during residency, or that of residents in other specialties. ${ }^{5}$ Probably more important than the setting, however, are the types of neurological problems encountered. Our data suggest that, despite the emphasis on in-patient time, there are surprising similarities between the case mix in residency and neurological practice, particularly with respect to the percentage of common disorders and non-neurological problems seen.

There are obvious limitations to comparing our data to surveys of practicing neurologists. In particular, we recorded new diagnoses, and not "clinical problems". For example, a patient entered as "Parkinson's disease" could be a newly diagnosed patient, a patient just starting treatment, an end-stage patient with motor fluctuations, or many other types of different clinical problems. It seems likely that the particular clinical problem associated with a given diagnosis would often be influenced by where the patient is seen, and in this respect the inpatient bias of residency training is likely to significantly influence its ability to prepare trainees for post-residency practice. In situations in which the clinical problem is diagnosis, especially for patients in the "no neurological diagnosis" category, the setting is probably less important. Confirmation of these speculations would require data different than ours, and could be an important direction for future research.

Four other potential weaknesses of our study can be noted. First, although we each recorded patient encounters prospectively, the study itself is retrospective, and we gathered data independently, without planning to analyze our data together. As a consequence, there may have been differences in how final diagnoses were assigned, although we do not think this is likely to be significant. Second, FGAM's log did not include the first nine months of his neurology training, although there is no reason to anticipate that a markedly different patient mix would have been seen during these months. Third, our data, along with that of D'Esposito, only represent the experiences of three residents in three programs, which may be too small a sample to allow confident generalization of the results. However, the similarity of our data sets is reassuring. Finally, we made no record of other patient encounters, such as follow-up care, which can certainly be an important part of residency training.

The parallels we have noted between our residency case mixes and the content of neurological practice should not be construed as an argument against change in residency training. Unquestionably there are many aspects of practice beyond a simple list of diagnoses for which current training programs provide inadequate preparation, and neurology training must adapt to changes in neurological practice. However, data such as ours are important to ensure that changes in training are based upon an accurate understanding of the experience provided by contemporary neurology training.

\section{REFERENCES}

1. Gelb DJ. Teaching neurology residents in the out-patient setting. Neurology 1994;51:817-820.

2. Ringel SP, Vickrey BG, Keran CM, Bieber J, Bradley WG. Training the future neurology workforce. Neurology 2000; 54: 480-484.

3. Desbiens R, Elleker MG, Goldsand G, et al. Current educational issues in the clinical neurosciences. Can J Neurol Sci 2001; 28: 299-308.

4. Corboy JR, Boudreau E, Morgenlander JC, Rudnicki S, Coyle PK. Neurology residency training at the millennium. Neurology 2002; 58: 1454-1460.

5. Schroeder SA, Showstack JA, Gerbert B. Residency training in internal medicine: time for a change? Ann Intern Med 1986; 104 : 554-561.

6. Wiest FC, Ferris TG, Gokhale M, et al. Preparedness of internal medicine and family practice residents for treating common conditions. JAMA 2002; 288: 2609-2614.

7. McLeod PJ, Snell L. Casemix in an internal medicine clerkship: Educational value of the clinical problems seen. J Gen Intern Med 1991; 6: 455-459.

8. D'Esposito M. Profile of a neurology residency. Arch Neurol 1995; 52: $1123-1126$.

9. Kurtzke JF. The current neurologic burden of illness and injury in the United States. Neurology 1982; 32: 1207-1214.

10. Simpson CA. A community neurologist's personal viewpoint on neurological training. Can. J. Neurol. Sci. 1977; 4: 265-268.

11. Murray TJ. Relevance in undergraduate neurology teaching. Can J Neurol Sci 1977; 4: 131-137.

12. MacDonald BK, Cockerell OC, Sander JWAS, Shorvon SD. The incidence and lifetime prevalence of neurological disorders in a prospective community-based study in the UK. Brain 2000; 123 : 665-676.

13. Ringel SP, Franklin GM, DeLapp C, Boyko EJ. A cross-sectional comparative study of outpatient neurologic practices in Colorado. Neurology 1988; 38: 1308-1314.

14. Menken M. The scope of neurologic practice. Evidence from a practice study. Arch Neurol 1985; 42: 386-387.

15. Perkin GD. Pattern of neurological outpatient practice: implications for undergraduate and postgraduate training. J R Soc Med 1986; 79: 655-657.

16. Rose AS. Graduate training in neurology. An assessment based on the opinions of 80 neurologists in private practice. Arch Neurol 1971; 24: 165-168. 


\section{APPENDIX}

Table 1. Neurologic disorders for which there were at least ten encounters during residency for FGAM.

\begin{tabular}{l|l}
\hline $\begin{array}{l}\text { Neurological disorder } \\
\text { Acute ischemic CVA/TIA }\end{array}$ & $\begin{array}{c}\text { Number }(\boldsymbol{\%}) \text { of patients } \\
(\mathbf{n}=\mathbf{1 0 9 4})\end{array}$ \\
\hline Epilepsy & $160(14.6)$ \\
\hline Headache & $143(13)$ \\
\hline Syncope & $114(10.4)$ \\
\hline Metabolic encephalopathy & $40(3.7)$ \\
\hline Vertigo (non CNS) & $37(3.4)$ \\
\hline Peripheral neuropathy & $34(3.1)$ \\
\hline Parkinson's disease & $34(3.1)$ \\
\hline Other movement disorders & $26(2.4)$ \\
\hline Multiple sclerosis & $23(2.1)$ \\
\hline Myopathy & $19(1.7)$ \\
\hline CNS infection & $19(1.7)$ \\
\hline Dementia & $18(1.6)$ \\
\hline Intracranial hemorrhage (not SAH) & $17(1.6)$ \\
\hline Closed head injury & $15(1.4)$ \\
\hline Brain/spinal metastasis & $13(1.2)$ \\
\hline Radiculopathy & $10(0.9)$ \\
\hline
\end{tabular}

$\mathrm{CNS}=$ central nervous system, $\mathrm{SAH}=$ sub arachnoid hemorrhage

Other diagnoses: Neurological symptoms without signs or diagnosis 175 (16.0), pediatric neurology diagnoses 63 (5.8) consult for prognosis 14 (1.3), psychiatric 13 (1.2), myelopathy $9(0.8)$, other neurodegenerative disease $8(0.7)$, Guillain-Barré syndrome 7 (0.6), SAH 7 (0.6), amyotrophic lateral sclerosis 6 (0.5), gait disorder $6(0.5)$, primary brain neoplasm $5(0.5)$, myasthenia gravis $5(0.5)$, ataxia $5(0.5)$, essential tremor $4(0.4)$, brain death $4(0.4)$, transient global amnesia $2(0.2)$, diabetes insipidis 1 (0.09), post-polio syndrome 1 (0.09), chemical meningitis 1 (0.09), hemifacial spasm 1 (0.09), pure autonomic failure 1 (0.09), Wernicke's encephalopathy 1 (0.09), endocarditis 1 (0.09), sarcoid 1 (0.09), vitamin B12 deficiency 1 (0.09), electrocution 1 (0.09), herpes zoster 1 (0.09), miscellaneous (including abnormal test result, pre-operative opinion, family history) 13 (1.2).
Table 2. Neurologic disorders for which there were at least 10 encounters during residency for $\mathrm{CC}$.

\begin{tabular}{|c|c|}
\hline Neurological disorder & $\begin{array}{c}\text { Number }(\%) \text { of patients } \\
(n=1009)\end{array}$ \\
\hline Acute ischemic CVA/TIA & $172(17.0)$ \\
\hline Peripheral neuropathy & $91(9.0)$ \\
\hline Radiculopathy & $84(8.3)$ \\
\hline Headache & $66(6.5)$ \\
\hline Epilepsy & $64(6.3)$ \\
\hline Primary brain neoplasm & $58(5.8)$ \\
\hline Metabolic encephalopathy & $41(4.1)$ \\
\hline Brain/spinal metastasis & $37(3.7)$ \\
\hline Other neurodegenerative disease & $35(3.5)$ \\
\hline Dementia & $32(3.2)$ \\
\hline Intracranial hemorrhage (not SAH) & $28(2.8)$ \\
\hline Parkinson's disease & $23(2.3)$ \\
\hline Vertigo (non CNS) & $20(2.0)$ \\
\hline CNS infection & $20(2.0)$ \\
\hline SAH & $19(1.9)$ \\
\hline Multiple sclerosis & $16(1.6)$ \\
\hline Syncope & $12(1.2)$ \\
\hline Amyotrophic lateral sclerosis & $12(1.2)$ \\
\hline Closed head injury & $10(1.0)$ \\
\hline Myopathy & $10(1.0)$ \\
\hline
\end{tabular}

CVA = cerobrovascular accident, TIA $=$ transient ischemic attack, $\mathrm{CNS}=$ central nervous system, $\mathrm{SAH}=$ sub-arachnoid hemorrhage

Other diagnoses: Neurological symptoms without signs or diagnosis $142(14.1)$, myasthenia gravis $7(0.7)$, sleep disorder 4 (0.4), essential tremor 4 (0.4), Guillain-Barré syndrome 2 (0.2). 